\section{BIRDS IN AND AROUND NANDED CITY, MAHARASHTRA}

\author{
A.N. Kulkarni ${ }^{1}$, V.S. Kanwate ${ }^{2}$ and V.D. Deshpande ${ }^{3}$ \\ 1,3 P.G. Department of Zoology, Science College, Nanded, \\ Maharashtra 431605, India \\ 2 Department of Zoology, V.N. College, Cidco, Nanded, Maharashtra \\ 431603, India \\ Email: 'ankulkarni12@rediffmail.com
}

Nanded district in South East of Maharashtra is situated on the bank of river Godavari. It has varied habitats like river, dams, lakes, mountain strips and agriculture fields. The predominating vegetation is typically dry deciduous type (Champion \& Jeth, 1968). Common flowering tree species are Acacia arabica, Melia azadirachta, Ficus religiosa, Mangifera indica, Ficus bengalensis, Tectona grandis, Zizyphus jujuba, Vitex negundo, Caesalpinia sp., Bombax sp., Millingtonia hortensis; Butea monosperma, Bauhinia racemosa, Eucalyptus sp., Terminalia panniculta. Dominant shrubs occurring here are Lantana camera, Ipomoea convoluta, Calotropis procera, Cassia sp. etc. Aquatic weeds like Hydrilla sp., Typha sp., Cyperus sp., Chara sp., Vallisneria sp., Pistia sp. etc. are present in water bodies.

Survey work by Yardi et al. (2004) conducted at Salim Ali lake Aurangabad, revealed the presence of 64 species of birds. Wadatkar and Kasambe (2002) reported 171 species of birds at Pohara-Malkhed Forest Reserve and Amaravati University Campus area, Amravati, Maharashtra. Avifauna at Nanded is unexplored as yet and hence the present survey work to record biodiversity of birds was conducted with scientific methodology that will form a base line information for future indepth studies.

The survey was carried out at six stations: 1. Osmannagar - as a road side station; 2. River Godavari - as a lotic habitat; 3. Science college campus - as a city area; 4 . Sita khandi - as a forest habitat; 5. Shikhachi Wadi - as a perennial lentic habitat; and 6. Derala Dam - as a semi perennial lentic and grass land habitat. These study stations are about $20-30 \mathrm{~km}$ from Nanded city except the science college, Nanded, which is situated in the heart of the city.

Methods: The reports is based on observation made from May 2000- May 2002. Regular visits were made to these study areas. Observations were carried on fixed path in $1 \mathrm{~km}$ radius in each station by using line transect method by Gaston (1973) for which nearly two hours were spent. The birds were sighted by using binoculars of $8 x$ and $7 x$ magnification and spot identification was done by using field guides (Ali \& Ripley, 1983; Ali, 1996; Grimmette et al., 1999; Kazmierczak, 2000; Manakadan \& Pittie, 2001) and only those species with confirmed identity are reported in this paper. A check list is prepared as per the guide lines given in the check list of birds (Abdulali, 1981; Gaikwad et al., 1997).
The status of the birds are catagorized as residential (R), migrant (M), resident migrant (RM), winter migrant (WM ), breeding migrant ( $\mathrm{BM}$ ) and passage migrant (PM). Abundance of birds was catagorised as common $(\mathrm{C})$, uncommon $(\mathrm{U})$, rare $(\mathrm{r})$ and occasional $(\mathrm{O})$.

Observations: The study reveals the occurance of 151 species of birds belonging to 44 families and 16 orders. Table 1 details the scientific and common names, status and occurrence of the birds. The list includes 86 species of resident birds, 32 species resident migrants, 19 species winter migrants, 10 species migrants, two species breeding migrants and two species passage migrants. Fifteen species, namely, Cattle Egret, Redwattled Lapwing, Blue Rock Pigeon, Eurasian collared-Dove, Rufous collared-Dove, Rose-ringed Parakeet, Greater Coucal, Brainfever Bird, House Swift, Small Bee-eater, Common Swallow, Black Drango, Common Myna, Red-vented Bulbul, Whitethroated Munia, were common in all the study stations. Maximum number of birds were recorded at Sitakhadi forest (81 species) where as only 46 species of birds were recorded at Science College Campus. Different types of aquatic birds were recorded at river Godavari, Sikhachiwadi, Sitakhandi and Derala. Local environmental conditions and season have impact on composition, diversity and occurrence of birds. Bird population was more during winter and summer. It was noted that birds moved out from one station to the other to avoid unfavorable environmental conditions. The Rosy Starling was found after winter season in flocks. Birds like Demoiselle Crane, Bar-headed Goose, White-bellied Heron, Asian Paradise-Flycatcher, Pied Crested Cuckoo were observed in very less numbers. Vultures and flamingos were not recorded during the span of study. The Comb Duck was observed only at Sitakhandi; Pheasanttailed Jacana was observed at Derala and Sitakhandi; while the Black Stork, a threatened bird was observed at Shikhachiwadi.

\section{REFERENCES}

Abdulali, H. (1981). Checklist of birds of Maharashtra. Bombay Natural History Society, Mumbai, 1-16pp.

Ali, S. (1996). The Book of Indian Birds. Bombay Natural History Society, Mumbai, 1-354pp.

Ali, S. and S.D. Ripley (1983). A Pictorial Guide to the Birds of the Indian Sub-continent. Bombay Natural History Society, Mumbai, 1-354pp.

Champion, H.G. and S.K. Seth (1968). A Revised survey of the forest types of India, Govt. of India, Delhi, 185pp.

Gaikwad, N., S. Puranik and N. Shah (1997). A check List of birds around

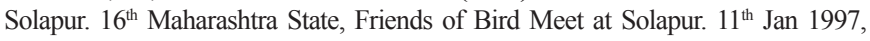
1-30pp (Unpublished)

Gaston, A.J. (1973). Methods for estimating bird populations. Journal of the Bombay Natural History Society 72(2): 272-281.

Grimmette, K., C. Inskipp and T. Inskipp (1999). Birds of Indian Sub continent. Oxford University Press, New Delhi, 384pp.

Karmierczak, K. (2000). A Field Guide to Birds of India. Pica Press, U.K., $351 \mathrm{pp}$.

Manakadan, R. and A. Pittie (2001). Standardised common and scientific names of the birds of the Indian subcontinant. Buceros 6(1): 1-37.

Wadatkar, J.S. (2001). Checklist of birds from Amravati Univrsity Campus, Amravati. Zoos' Print Journal 16(5): 497-499.

Wadatkar, J.S. and R. Kasambe (2002). Checklist of birds from PoharaMalkhed reserve forest, Dist. Amaravati, Maharashtra. Zoos' Print Journal 17(6): 807-811.

Yardi, D., S.S Patil and R.G. Auti (2004). Diversity of Avian fauna from salim Ali Lake at Aurangabad. Paper presented in $21^{\text {st }}$ meet of Bird lovers of Maharashtra held at Nanded on $3^{\text {rd }} \& 4^{\text {th }}$ April 2004 (Unpublished). 
Table 1. Checklist of birds in and around Nanded city, Maharashtra

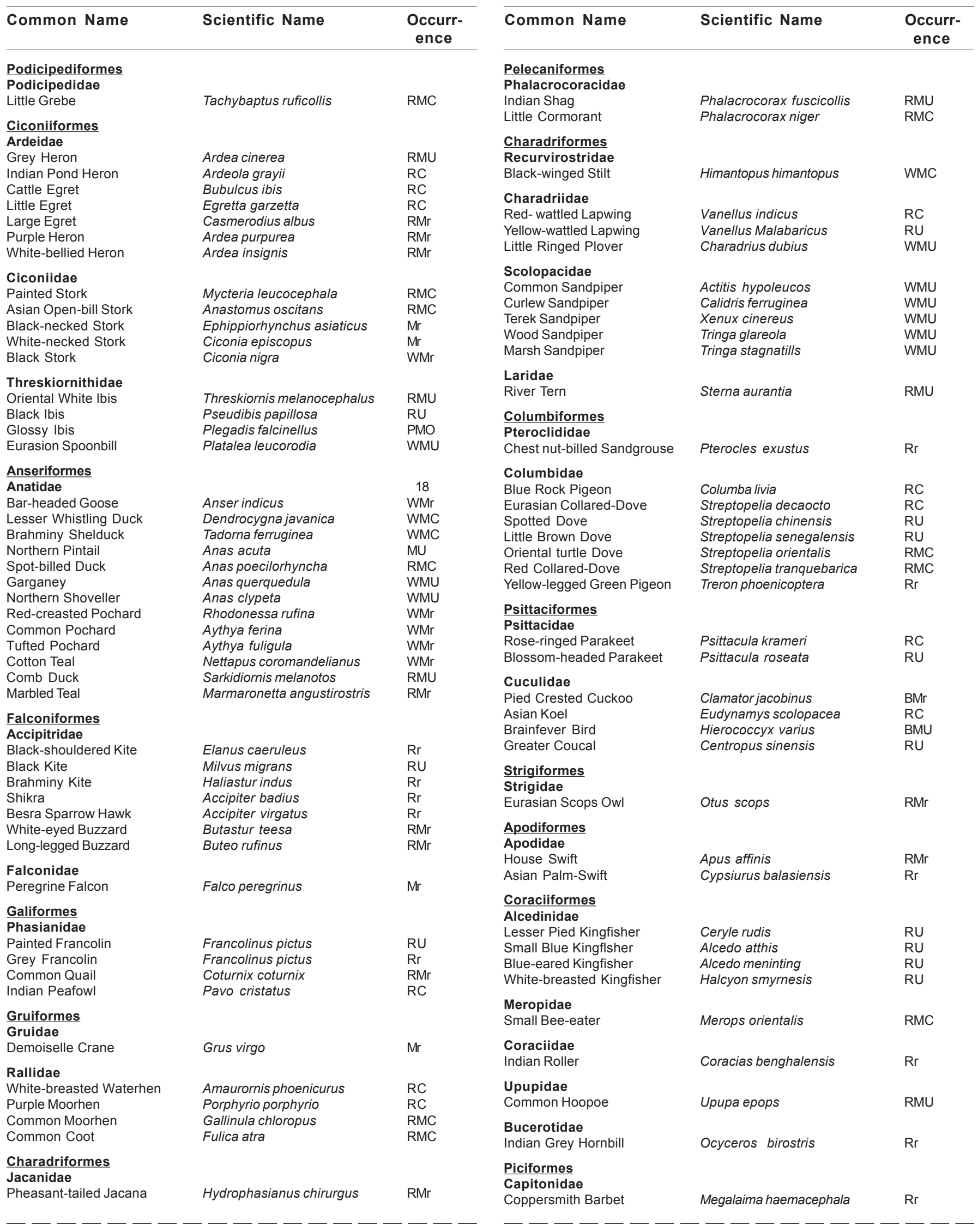




\begin{tabular}{|c|c|c|c|c|c|}
\hline Common Name & Scientific Name & $\begin{array}{l}\text { Occurr- } \\
\text { ence }\end{array}$ & Common Name & Scientific Name & $\begin{array}{l}\text { Occurr- } \\
\text { ence }\end{array}$ \\
\hline \multicolumn{3}{|l|}{ Picidae } & Yellow Wagtail & Motacilla flava & WMC \\
\hline \multirow{2}{*}{\multicolumn{3}{|c|}{ Passeriformes }} & Grey Wagtail & Motacilla cinerea & $\mathrm{Mr}$ \\
\hline & & & White Wagtail & Motacilla alba & MU \\
\hline \multicolumn{3}{|l|}{$\begin{array}{l}\text { Passeritormes } \\
\text { Alaudidae }\end{array}$} & Large Pied Wagtail & Motacilla maderaspatensis & MU \\
\hline Rufous-tailed FinchLark & Ammomanes phoenisurus & $\mathrm{RU}$ & Forest Wagtail & Dendronanthus indicus & $\mathrm{RM}$ \\
\hline Greater Short- toed Lark & Calandrella brachydactyla & RU & \multicolumn{3}{|l|}{ Nectariniidae } \\
\hline Indian Short-toed Lark & Calandrella raytal & $\mathrm{MR}$ & Purple-rumped Sunbird & Nectarinia zeylonica & $\mathrm{RU}$ \\
\hline Black-crowned Sparrow-Lark & Eremopterix nigriceps & RU & Purple Sunbird & Nectarinia asiatica & $\mathrm{Rr}$ \\
\hline \multirow{2}{*}{$\begin{array}{l}\text { Bengal Bush-Lark } \\
\text { Eurasian Skylark }\end{array}$} & Mirafra assamica & RU & Small Sunbird & Nectarinia minima & $\mathrm{RU}$ \\
\hline & Alauda arvensis & $\mathrm{RU}$ & \multicolumn{3}{|l|}{ Zosteropidae } \\
\hline \multicolumn{3}{|l|}{ Hirundinidae } & Oriental White-eye & Zosterops palpebrosus & $\mathrm{RU}$ \\
\hline \multirow{5}{*}{$\begin{array}{l}\text { Wire-tailed Swallow } \\
\text { Common Swallow } \\
\text { House Swallow } \\
\text { Red-rumped Swallow } \\
\text { Dusky Crag-martin } \\
\text { Streak-throated Swallow }\end{array}$} & $\begin{array}{l}\text { Hirundo smithii } \\
\text { Hirundo rustica }\end{array}$ & $\begin{array}{l}\mathrm{RU} \\
\mathrm{RMC}\end{array}$ & \multicolumn{3}{|l|}{ Passeridae } \\
\hline & Hirundo tahitica & $\mathrm{RC}$ & Passerinae & & \\
\hline & Hirndo daurica & RMC & House Sparrow & Passer domesticus & $\mathrm{RC}$ \\
\hline & Hirundo concolor & $\mathrm{RC}$ & \multicolumn{3}{|l|}{ Ploceinae } \\
\hline & Hirundo fluvicola & $\mathrm{RU}$ & Baya Weaver & Ploceus philippinus & $\mathrm{RC}$ \\
\hline \multicolumn{3}{|l|}{ Laniidae } & \multicolumn{3}{|l|}{ Estrildidae } \\
\hline Great Grey Shrike & Lanius excubitor & RU & White-throated Munia & Lonchura malabarica & $\mathrm{RC}$ \\
\hline Baybacked Shrike & Lanius vittatus & RU & Spotted Munia & Lonchura punctulata & RU \\
\hline Rufous-backed Shrike & Lanius schach & $\mathrm{RU}$ & Black-throated Munia & Lonchura kelaarti & $\mathrm{RU}$ \\
\hline \multicolumn{3}{|l|}{ Dicruridae } & \multicolumn{3}{|l|}{ Campephagidae } \\
\hline Black Drongo & Dicrurus macrocercus & $\mathrm{RC}$ & Rosy Minivet & Pericrocotus roseus & $\mathrm{Rr}$ \\
\hline White-bellied Drongo & Dicrurus caerulescens & $\mathrm{RU}$ & Scarlet Minivet & Pericrocotus flammeus & $\mathrm{Rr}$ \\
\hline \multicolumn{3}{|l|}{ Sturnidae } & Pied Flycatcher Shrike & Hemipus-picatus & $\mathrm{Rr}$ \\
\hline Common Myna & Acridotheres tristis & $\mathrm{RC}$ & Common Wood Shrike & Tephrodornis pondicerianus & $\mathrm{RU}$ \\
\hline Brahminy Starling & Sturnus pagodarum & $\mathrm{RC}$ & & & \\
\hline Asian Pied Starling & Sturnus contra & RU & RMC - Resident Migrant & רon; RMU - Resident Migrant & ommon; \\
\hline Rosy Starling & Sturnus roseus & PMC & RC - Resident Common & Resident Migrant rare; M - M & \\
\hline Corvidae & & & $\begin{array}{l}\text { Mr - Migrant rare; WMr } \\
\text { PMO - Passage Migrant }\end{array}$ & $\begin{aligned} & r \text { Migrant rare; } R U \text { - Resident } \\
& \text { ional: PMC - Passage Migrant }\end{aligned}$ & $\begin{array}{l}\text { ommon; } \\
\text { nmon: }\end{array}$ \\
\hline Indian Treepie & Dendrocitta vagabunda & RU & WMU - Winter Migrant L & non: WMC - Winter Miarant C & hon; \\
\hline House Crow & Corvus splendens & RC & MU - Migrant Uncommo & Resident rare; BMr - Breeding & rant rare; \\
\hline Jungle Crow & Corvus macrorhynchos & RU & BMU - Breeding Migrant & $\begin{array}{l}\text { nmon } \\
\text { ant - Dreeding }\end{array}$ & rall tale, \\
\hline Irenidae & & & & & \\
\hline Common lora & Aegithina tiphia & RU & & & \\
\hline Gold-fronted Chloropsis & Chloropsis aurifrons & $\mathrm{Rr}$ & ACKNOWLEDGEMEN? & & \\
\hline $\begin{array}{l}\text { Pycnonotidae } \\
\text { Red-vented Bulbul }\end{array}$ & Pycnonotus cafer & $\mathrm{RC}$ & $\begin{array}{l}\text { Authors are thankful to } \\
\text { financial assistance to }\end{array}$ & $\begin{array}{l}\text { (Western Regional Center, Pu } \\
\text { ut the minor research project }\end{array}$ & $\begin{array}{l}\text { for providing } \\
\text { le record our }\end{array}$ \\
\hline Black Bulbul & Hypsipetes leucocephalus & $\mathrm{Rr}$ & thanks to principal, Scier & lege, Nanded for constant en & ggement. We \\
\hline $\begin{array}{l}\text { Muscicapidae } \\
\text { Timaliinae }\end{array}$ & & & $\begin{array}{l}\text { are thankful to experts } \\
\text { Pune) for guidance. }\end{array}$ & NHS Mumbai and ZSI Pune & stern Region, \\
\hline Large Grey Babbler & Turdoides malcolmi & $\mathrm{RC}$ & & & \\
\hline Jungle Babbler & Turdoides striatus & $\mathrm{RC}$ & & & \\
\hline Yellow-eyed Babbler & Chrysomma sinense & $\mathrm{Rr}$ & & & \\
\hline Yellow-breasted Babbler & Macronous gularis & $\mathrm{Rr}$ & & & \\
\hline Muscicapinae & & & & & \\
\hline Little Pied Flycatcher & Ficedula westermanni & $\mathrm{Rr}$ & & & \\
\hline Asian Brown Flycatcher & Muscicapa dauurica & $\mathrm{RMr}$ & & & \\
\hline Monarchinae & & & & & \\
\hline Asian Paradise-Flycatcher & Terpsiphone paradisi & $\mathrm{RMr}$ & & & \\
\hline Sylviinae & & & & & \\
\hline Plain Prinia & Prinia inornata & RU & & & \\
\hline Ashy Prinia & Prinia socialis & RU & & & \\
\hline Jungle Prinia & Prinia sylvatica & RU & & & \\
\hline Rufus-fronted Prinia & Prinia buchanani & RU & & & \\
\hline Common Tailor Bird & Orthotomus sutorius & $\mathrm{Rr}$ & & & \\
\hline Thick-billed Warbler & Acrocephalus aedon & $\mathrm{Mr}$ & & & \\
\hline $\begin{array}{l}\text { Turdinae } \\
\text { Indian Robin }\end{array}$ & & & & & \\
\hline Indian Robin & Saxicoloides fulicata & RU & & & \\
\hline Indian Chat & Cercomela fusca & $R U$ & & & \\
\hline Paridae & & & & & \\
\hline Great Tit & Parus major & $\mathrm{Rr}$ & & & \\
\hline Pied Tit & Parus nuchalis & $\mathrm{Rr}$ & & & \\
\hline Motacillidae & & & & & \\
\hline Oriental Tree Pipit & Anthus hodgsoni & $\mathrm{RMr}$ & & & \\
\hline
\end{tabular}

